\title{
Successful management without protective colostomy in an adult patient with anorectal malformation
}

\author{
Cengiz Tavusbay', Hüdai Genç ${ }^{1}$, Irfan Karaca², Kemal Atahan¹, Mehmet Hacıyanlı1, Erdal Türk²
}

\begin{abstract}
Anorectal malformations are rare occurrences characterized by the absence or abnormal localization of the anus. Clinical manifestations can vary from mild forms that require only minor surgery to more complicated cases that must be managed with multi-staged surgery. In this report, our aim is to present the clinical characteristics, management, and treatment outcome of an adult patient with an anorectal malformation with a vestibular fistula that was successfully repaired by posterior sagittal anorectoplasty (PSARP) and to discuss the case in the light of the relevant literature. We also would like to underline the fact that primary anorectal malformations can, although rarely, be observed in adult patients. A 26-year-old female patient presented with an imperforate anus and constipation with feces passing through a fistula located at the distal part of her vagina. She was continent for solid feces, but had leakage of flatus and fecal soiling. She also had concurrent left renal agenesis. A posterior sagittal anorectoplasty was successfully performed without a colostomy. The patient was discharged uneventfully on the postoperative 8th day. The patient was fully continent during a control visit after seven years of follow-up. Posterior sagittal anorectoplasty is a technique that can be successfully used in adult patients with anorectal malformations.

Keywords: Anorectal malformation, colostomy, imperforate anus, posterior sagittal anorectoplasty, rectovestibular fistula
\end{abstract}

Cite this paper as:

Tavusbay C, Genç H, Karaca I, Atahan K, Haclyanlı M, Türk

E. Successful management

without protective colostomy

in an adult patient with

anorectal malformation. Turk

J Surg 2017; 33: 205-208.

'Department of General Surgery, İzmir Katip Çelebi University Atatürk Training and Research Hospital, İzmir, Turkey ${ }^{2}$ Department of Pediatric Surgery, Medical Park Izmir Hospital, İzmir, Turkey

Address for Correspondence Cengiz Tavusbay e-mail:tavusbay3@gmail.com

Received: 07.05.2015 Accepted: 29.08.2015

\section{OCopyright 2017} by Turkish Surgical Association

Available online at www.turkjsurg.com

\section{INTRODUCTION}

Anorectal malformations (ARM) are embryonic developmental disorders characterized by an absence of the anus or the rectum opening to an abnormal anatomical location. Embryologically, the condition is caused by a developmental disorder of the cloaca and the transverse septum (urorectal septum), which separates the gastrointestinal system from the urogenital system. In the literature, the condition has been reported with a prevalence of 1 in 1500 to 5000 births $(1,2)$. However, different prevalences are observed in different parts of the world. In female children, a fistula usually develops into the vulva, vagina, urethra, perineum, or vestibule. In male children, the fistula develops into the scrotum or the urethra.

In 1982, De Vries and Pena described posterior sagittal anorectoplasty (PSARP) for the treatment of anorectal malformations $(2,3)$. Since that time, this technique has become widely accepted in the surgical treatment of anorectal malformations and is now the gold standard (4). Today, this technique is used to treat anorectal malformations observed both in neonates and pediatric patients as well as, rarely, in adults.

In this report, we would like to present a patient who was diagnosed with an anorectal malformation in adulthood and was successfully treated through surgery in light of the relevant literature.

\section{CASE PRESENTATION}

A 26-year-old female patient with mild mental retardation presented with constant constipation, gas, and defecation through the vagina. The patient, who did not seek medical assistance until this age for various reasons (family negligence, social factors, and low level of education), was brought to our outpatient clinic by her relatives.

Her physical examination revealed a distinctive developmental retardation. Her height was $137 \mathrm{~cm}$, and she weighed $37 \mathrm{~kg}$. She also had mild mental retardation. No pathologies were observed in her systemic examination. The perineal exam revealed an anorectal malformation with a retrovestibular fistula, and the anal canal ended in front of the hymen. During the preoperative period, the patient underwent intravenous pyelography (IVP), complete abdominal ultrasonography (USG), magnetic resonance imaging (MRI) of the whole abdomen and pelvic region, electromyography (EMG) of the perianal muscles, and barium graphy of the colon. In addition to the diagnosed anomaly, the tests revealed a left renal agenesis in the patient. The barium graphy indicated a dilatation of the sigmoid colon. Based on these 


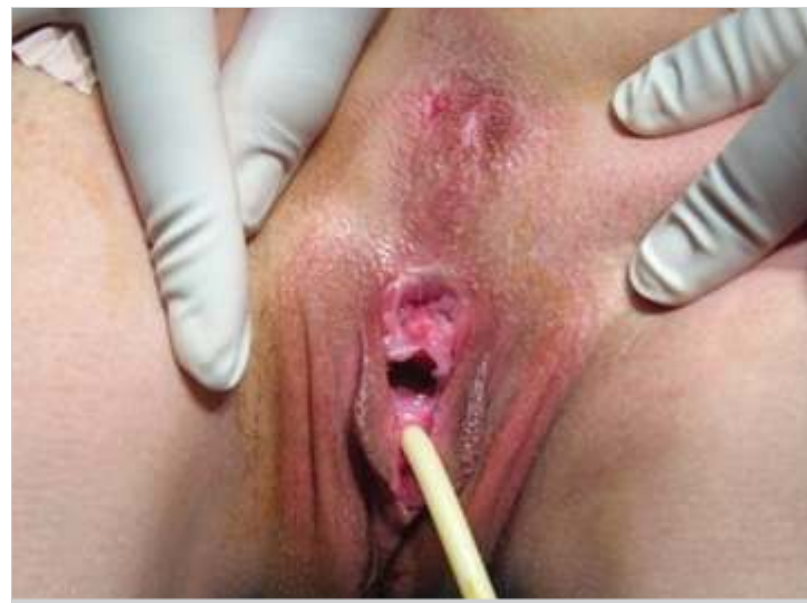

Figure 1. Preoperative view of the patient. The absence of the anal orifice is obvious

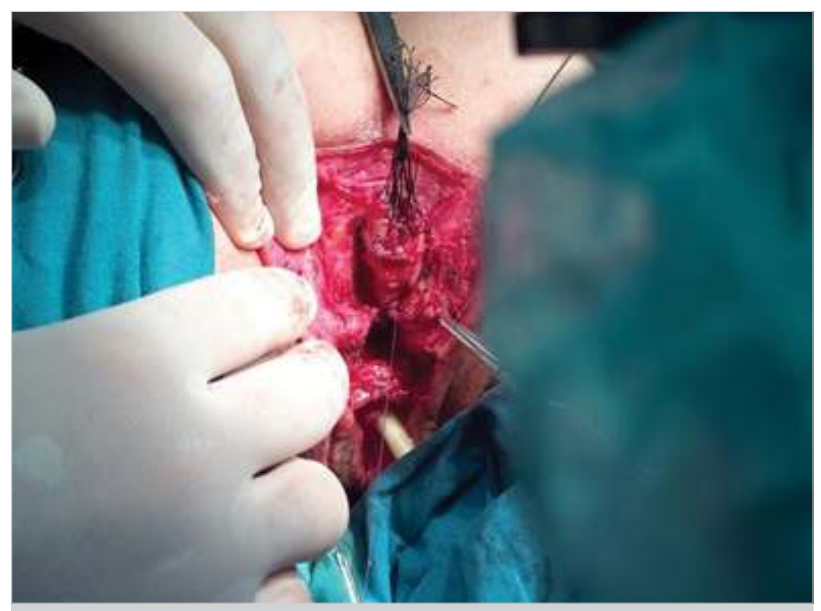

Figure 3. The anterior muscle mass and construction of the anterior commissure

findings, the patient was prescribed enema and oral liquid paraffin for six months (Sokol liquid; Munir Sahin llac, İstanbul, Turkey).

\section{SURGICAL TECHNIQUE}

The patient was referred for pediatric surgery, urology, gynecology, psychology, and neurology consultations. Following presurgical colon preparation, under general anesthesia, a Foley catheter was placed, and the patient was brought to the jackknife position (Figure 1). Using 3/0 atraumatic polyglactin 910 (Vicryl; Ethicon, Summerville, NJ, USA), suspension sutures at 3 to $4 \mathrm{~mm}$ intervals were made at the mucocutaneous junction of the anus. Then, a vertical incision was made through the layers from the lower end of the sacrum to the commisura posterior over the rectovestibular fistula. The external sphincter and the muscle complex were prepared until the rectum was located in the midline (Figure 2,3). Equal amounts of muscle were left on both sides. The rectum was separated from the vagina up to the peritoneal reflexion. After the rectum was fully released, the perineal body was repaired using $2 / 0$ polyglactin 910 (Vicryl; Ethicon, Summerville, NJ, USA) sutures (Figure 4). First, the anterior part of the muscle group was brought together, followed by the posterior part (Figure 5). The midline of the skin was closed, and the anoplasty was completed by su-

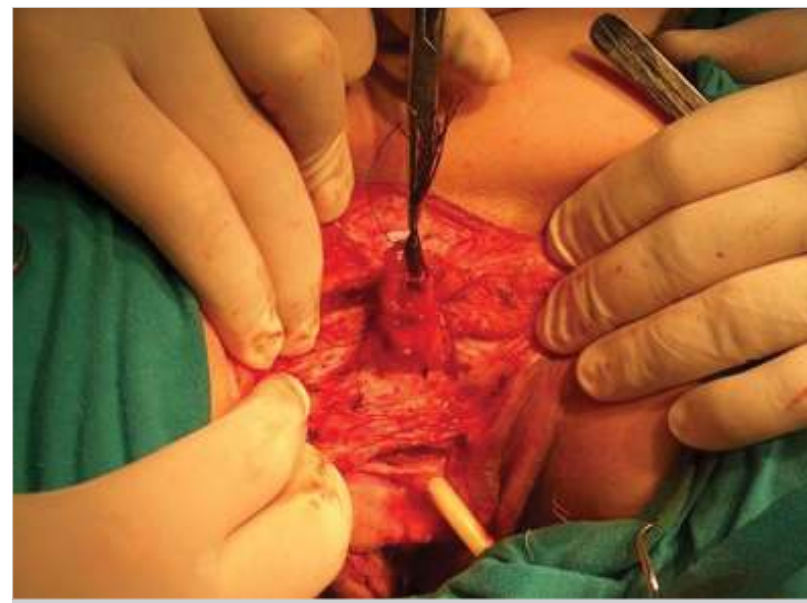

Figure 2. The rectovestibular fistula is fully revealed. The vagina is easily visible below it

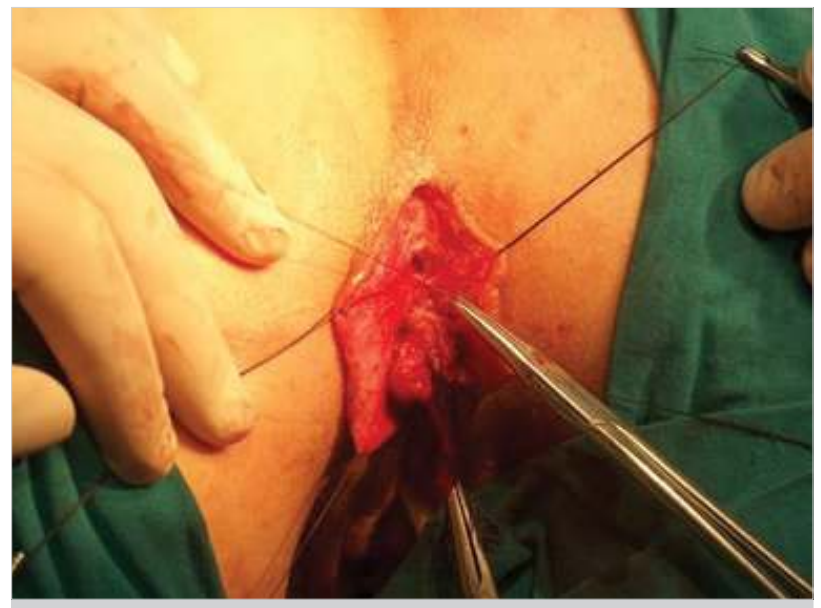

Figure 4. Repair of the posterior muscle complex

turing the rectal section to be anastomized to the skin so that it remained within the borders of the external sphincter (Figure 6). During the postoperative period, the patient was not fed orally for the first week. Because no complications developed, the patient was discharged on the $10^{\text {th }}$ postoperative day.

The patient was followed up for seven years. In her last control visit, she stated that she defecates regularly once a day and maintains anal continence. Her physical examination revealed no abnormalities (Figure 7). The patient did not develop anal incontinence during her seven-year follow-up.

\section{DISCUSSION}

Anorectal malformations include a number of congenital defects and various types of urinary and/or sexual malformations. A patient with an anorectal anomaly seeking medical assistance and treatment for the first time in adulthood is a rare occurrence $(1,5)$. These patients are usually diagnosed and treated during infancy and childhood. Although anorectal malformations usually occur as an isolated anomaly, they may also be accompanied by one or more anomalies affecting other organs. Therefore, in both pediatric and adult patients, the whole system of a patient with anorectal malformations should be very carefully examined. 


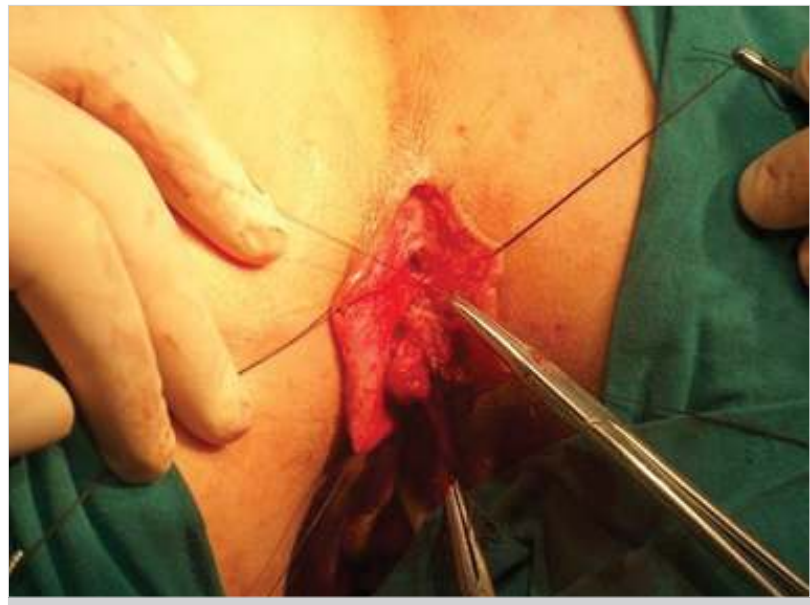

Figure 5. Closure of the skin and fixation of the anus to the skin

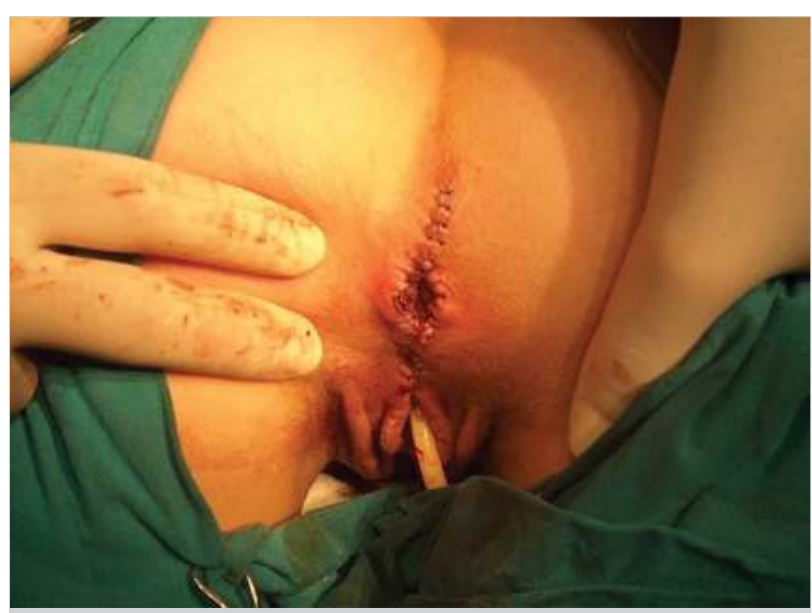

Figure 6. Postsurgical view

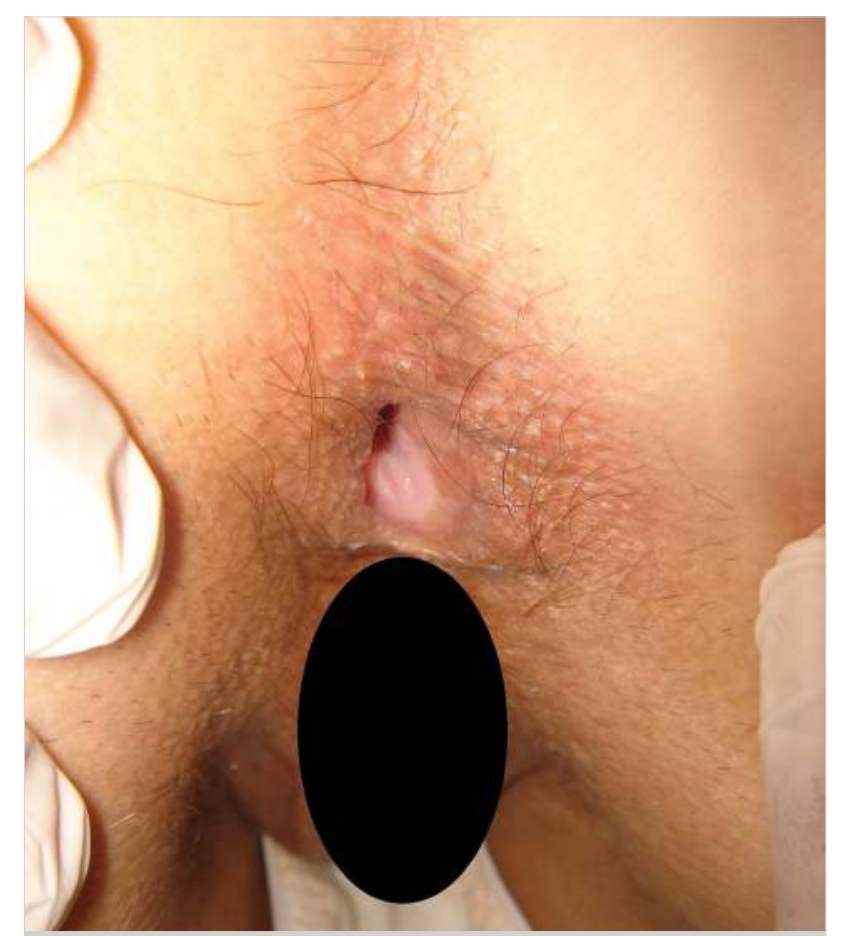

Figure 7. One-year follow-up of the patient
Table 1. Krickenbeck classification of ARM

\begin{tabular}{|ll|}
\hline I. Major clinical groups & II. Rare/regional variants \\
\hline 1. Perineal (cutaneous) fistula & 1. Pouch colon atresia/stenosis \\
\hline 2. Rectourethral fistula: & 2. Rectal atresia/stenosis \\
\hline i. Prostatic & 3. Rectovaginal fistula \\
\hline ii. Bulbar & 4. H-type fistula \\
\hline 3. Rectovesical fistula & 5. Others \\
\hline 4. Vestibular fistula & \\
\hline 5. Cloaca & \\
\hline
\end{tabular}

6. ARMs with no fistula

\section{Anal stenosis}

ARM: anorectal malformations

In adult patients, to determine the ideal treatment option and to achieve a satisfactory surgical result, the type and size of the defect should be carefully examined, and the anatomic structure and borders of the perineum and pelvis should be examined in detail. Also, the patient's defecation habits, which may involve constant diarrhea or constipation, are of great importance. Patients with constipation are reported to respond better to bowel training (4). Our patient also complained of constipation during the preoperative period, and barium graphy of the colon revealed a dilated rectum and sigmoid colon. To normalize the colonic calibration and regulate the intestinal function, the patient was administered an oral fluid and an enema through the fistula to soften the feces. Thus, the diameter of the sigmoid colon was brought closer to the normal size.

In all patients, direct X-rays, MCUG, fistulography, and computed tomography of the whole abdomen, MRI, USG, and colonography with a water-soluble contrast agent should be performed to detect concurrent anomalies (6). Especially, the detection of the contours of the sigmoid colon, colonic dilatation, and the length of the colon segment to be used for the repair are highly important. Even when the patient does not have any complaints related to the urinary system, the urinary system should be examined in detail in all patients. In our patient, left kidney agenesis was also detected. Pelvic MRI is important in determining anatomical differences, especially the relationship of the rectum and the sphincter. These imaging methods also reveal any additional pathologies, such as dermoid cysts or other benign tumors (7).

Although various classifications are used for this purpose, in a symposium held to resolve terminological confusion on the international level, the Krickenbeck classification presented in Table 1 gained prominence (2). According to this classification, our patient was defined as vestibular type in the major clinical group. The vestibular fistula in our patient is the most commonly observed anorectal defect in female pediatric patients with anorectal malformations (2).

Postsurgical prognosis is better in adult patients in whom the gluteal cleft is fully developed beside the sacrum and the normal gluteal muscle mass, the nerves are intact to maintain the anal reflex, and the voluntary perineal muscles are visibly con- 
tractile (3). In our patient, preoperative tests revealed these structures and the anocutaneous reflex to be normal. In the literature, PSARP has been reported to be a successful method with satisfactory results in the rare adult patients with anorectal malformations (1-5). The crucial part of this surgical technique is the dissection and separation of the rectovaginal wall at full thickness. The decision to perform a colostomy should be made after meticulous consideration in adults. A colostomy may not be necessary in adult patients with optimal passages or in whom the passage is maintained at the desired level through medical methods. Thus, PSARP without a colostomy may achieve satisfactory results in selected patients. In the present patient, PSARP was performed without a colostomy, which led to an excellent result.

\section{CONCLUSION}

Anorectal malformations may be rarely observed in adulthood. In selected adult patients carefully examined in the preoperative period, the PSARP method may be successfully applied without a colostomy. In these patients, concomitant anomalies should be considered and the necessary tests should be performed. It is also important to observe the size and type of the lesion. A comprehensive preoperative evaluation will doubtlessly influence the surgical outcome. Thus, we believe that other departments, including mainly pediatric surgery, gynecology, and urology, should be involved in the systemic evaluation of adult patients with anorectal malformations.

Informed Consent: Written informed consent was obtained from patient who participated in this study.

Peer-review: Externally peer-reviewed.
Contributions: Concept - C.T., I.K., H.G., E.T.; Design - C.T., I.K., M.H.; Supervision - C.T., I.K., K.A.; Resource - I.K., H.G.; Materials - H.G., I.K., M.H., E.T.; Data Collection and/or Processing - C.T., I.K., H.G.; Analysis and/or Interpretation - C.T., I.K., K.A., M.H.; Literature Search - C.T., K.T., M.H., I.K., E.T.; Writing Manuscript - C.T., I.K., H.G.; Critical Reviews - C.T., I.K., M.H., K.A, E.T.

Conflict of Interest: No conflict of interest was declared by the authors.

Financial Disclosure: The authors declared that this study has received no financial support.

\section{REFERENCES}

1. Terzi A, Coskun A, Yildiz F, Coban S, Akinci OF. Anovestibular fistula with imperforate anus in two adults. Ann Saudi Med 2008; 28: 472-474. [CrossRef]

2. Murphy F, Puri P, Hutson J, Holschneider A. Incidence and frequency of different types and classification of anorectal malformations. In: Holschneider AM, Hutson JM (Eds.) Anorectal malformations in children. Springer-Verlag Berlin Heidelberg; 2006. p. 161-184.

3. deVries PA, Peña A. Posterior sagittal anorectoplasty. J Pediatr Surg 1982; 17: 638-643. [CrossRef]

4. Simmang CL, Huber PJ Jr, Guzzetta P, Crockett J, Martinez R. Posterior sagittal anorectop-lasty in adults: secondary repair for persistent incontinence in patients with anorectal malforma-tions. Dis Colon Rectum 1999; 42: 1022-1027. [CrossRef]

5. Duman K, Ozdemir Y, Yigitler C, Gulec B. Rectocutaneous fistula with imperforate anus in an adult. Singapore Med J 2013; 54: 85-87. [CrossRef]

6. Patrapinyokul S, Sujijantararat P. Posterior sagittal anorectoplasty for imperforate anus: a pre-liminary result. J Med Assoc Thai 1993; 76: 23-28.

7. Tsugawa C, Hisano K, Nishijima E, Muraji T, Satoh S. Posterior sagittal anorectoplasty for failed imperforate anus surgery: lesson slearned from secondary repairs. J Pediatr Surg 2000; 35: 16261629. [CrossRef] 